\title{
A Study on Microscopic Simulation of Roundabout's Capacity
}

\author{
Xu Luwei, Liao Hui, Huang Chonglin
}

Guangdong University of Petrochemical Technology, Maoming, China

\section{Keywords: VISSIM; roundabout; weight calculation}

Abstract: The capacity of roundabout by using the simulation software VISSIM, which mainly focuses on the use of this software. By using the method of weight calculation, the factors of affecting the capacity of roundabout are concluded, and at last, through modeling and simulating the roundabout in Maoming, the analysis results are reached just as the physical truth.

\section{Introduction}

Roundabout is a special form of intersection, which commonly used in heavy traffic areas in cities and towns. By setting a roundabout in the center of it, the vehicles can be organized into the roundabout, and drive around it in a counter-clockwise direction. Through the motion of shunting, interleaving, and the confluence of vehicles, the traffic conflict points are turned into intertwined points, which reduce the severity of vehicle conflicts. The application of it not only improves the security rate of vehicle, but also makes the vehicle run smoothly. But some special situations may appear. For example, when the flow of each single inlet or outlet outweighs its inherent capacity, the roundabout shows impediment to the flow of traffic, causing traffic jams easily or even locking phenomenon. Thus the study on microscopic simulation of roundabout's capacity is particularly important.

Roundabout has been used in China's urban road in a early time, and plenty of scholars have studied it. But most scholars mainly investigate the effects of vehicle's flow to intersection's capacity, ignoring the important influence of non-motor vehicles on roundabout. With the rapid development of electric vehicles in recent years, the patterns of road and vehicle have been reclassified, and the proportion of those cars requires us to optimize the model of roundabout.

\section{The Process of Establishing Simulation Model}

When configuring the annular intersections, firstly, select the editing control signal button of signal control on the topmost menu bar. After a new signal timing scheme created, select the simplest fixed timing to set up. Fig. 1 is an example of signal timing scheme.

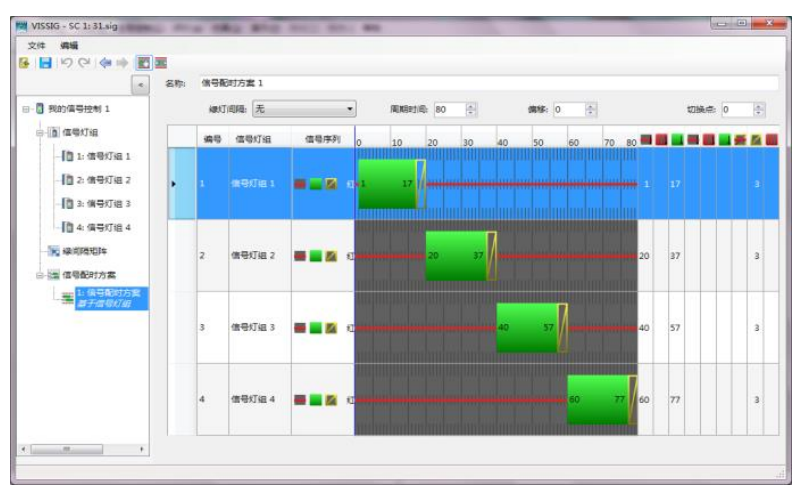

Fig.1 Signal timing scheme 
Thereafter, some unresolved conflicts contained in the signal lights of the intersection can be dealt in conflict zones, and then the effect with all the conflicts is resolved, which is shown in Fig. 2.

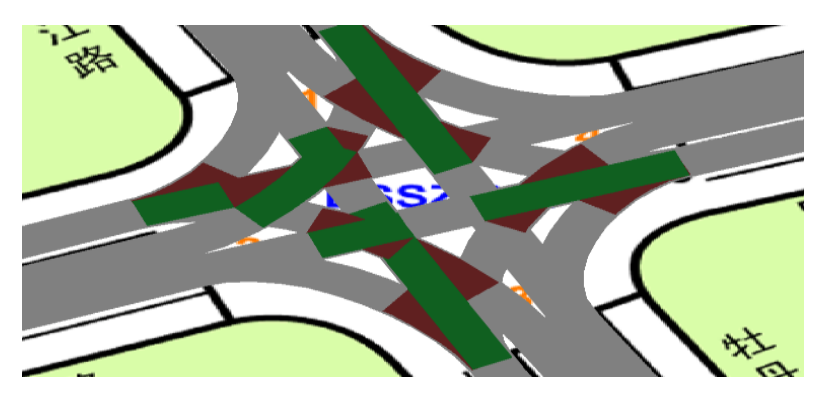

Fig.2 The treatment effect in the conflict zone

\section{Study of Factors Affecting Roundabout's Running}

In this paper, the analytic model is a roundabout that has four branches with no signal controlled. To make the outputs of simulation more representatives, here specially making the same proportion between geometric conditions of those four branches and the flow as well as the steering ratio inputted. the data and simulating road networking shown as Tab.1.

Tab1. Geometric dimension of simulating analytic model

\begin{tabular}{cc}
\hline Name & Value \\
\hline Radius of roundabout & $50[\mathrm{~m}]$ \\
Lanes in roundabout & 2 \\
Number of lanes in each inlet & 2 \\
Number of lanes in each outlet & 2 \\
Lane length & $3.5[\mathrm{~m}]$ \\
\hline
\end{tabular}

Through the principle of Given a proposed two, separately changing those affecting factors like the average parking distance, additional portion of safety distance, multiple portion of safety distance and acceptable deceleration. Meanwhile, when keeping other factors unchanged, and then we can use VISSIM to calculate their weight values.

\section{Case Application}

\subsection{Operating Characteristic of Roundabout in the Example.}

This paper mainly analyzes the roundabout in Maoming city, and its running states is like Fig. 3. 


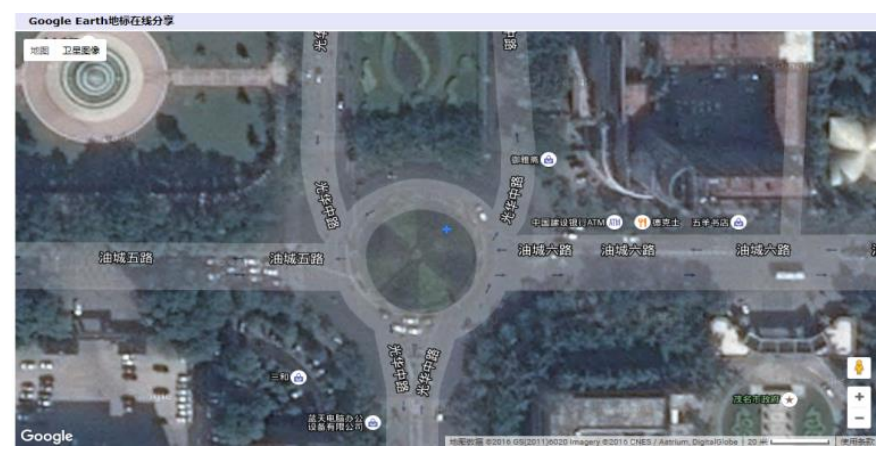

Fig 3. Satellite imagery of roundabout

\subsection{Investigation Data of Roundabout in the Example.}

In this investigation to the roundabout, many methods have been combined: high angle shot with unmanned aerial video, filed investigation, filed measurement, and data collecting shown below in Tab.2.

Tab.2. Geometric conditions of roundabout

\begin{tabular}{cccc}
\hline Name & Number of lanes & Width of lanes & Radius \\
\hline Ring inside lane and roundabout & 2 lanes & $4.0[\mathrm{~m}]$ & $30[\mathrm{~m}]$ \\
East inlet & Two-way four lanes & $4.0[\mathrm{~m}]$ & - \\
South inlet & Two-way four lanes & $3.5[\mathrm{~m}]$ & - \\
West inlet & Two-way four lane & $3.5[\mathrm{~m}]$ & - \\
North inlet & Independent two-way four lane & $4.5[\mathrm{~m}]$ & - \\
\hline
\end{tabular}

\subsection{Establishing of Simulation Model and Calculation of Intersection's Capacity.}

Through the actual monitoring data, we can detect the average delay of operational vehicles in different period in roundabout by using VISSIM. This is the output of each flow and average delay in different time, shown in Tab.3. 
Tab.3. Data of flows and average delay in different time

\begin{tabular}{ccccc}
\hline Period & $\begin{array}{c}\text { Flow of vehicles } \\
{[\mathrm{pcu} / \mathrm{h}]}\end{array}$ & $\begin{array}{c}\text { Flow of non-motor vehicle } \\
{[\mathrm{pcu} / \mathrm{h}]}\end{array}$ & $\begin{array}{c}\text { Total flow } \\
{[\mathrm{pcu} / \mathrm{h}]}\end{array}$ & $\begin{array}{c}\text { Average delay } \\
{[\mathrm{s}]}\end{array}$ \\
\hline 1 & 1800 & 1605 & 3405 & 1.1 \\
2 & 1800 & 1605 & 3405 & 4.1 \\
3 & 2200 & 1605 & 3805 & 8.1 \\
4 & 2400 & 1605 & 4005 & 10.3 \\
5 & 2600 & 1605 & 4205 & 16.8 \\
6 & 2800 & 1605 & 4405 & 19.1 \\
7 & 3000 & 1605 & 4605 & 28.2 \\
8 & 3200 & 1605 & 4805 & 45.6 \\
9 & 3400 & 1605 & 5005 & 112.8 \\
\hline
\end{tabular}

From the results of delay in Tab.3, it is easier to know that delay time in period 8 and 9 changes dramatically. According to the criteria of classifying service level in China's non-signalized intersection, at this time, the traffic is in congestion, which causes big delay. And that is the reason why the actual capacity of this roundabout is the sum of flows of vehicles and non-motor vehicles in period 8 , which is $4805 \mathrm{pcu} / \mathrm{h}$.

\section{Conclusions}

This paper mainly applies the simulation software VISSIM to establish and analyze the roundabout model, introducing the process of building simulation model. In the process, data simulation and practical calculation are combined to ensure preciseness. Making use of the principle of given a proposed twoto study the effect that various evaluation parameters have made on the maximum queuing length and running time when operating the software VISSIM. Finally, we take the roundabout in Guanghua-Guandu Road Maoming City as an example, calculating and analyzing its capacity. During the process, considering the non-motor vehicles account for a large proportion of flows, we calculate it and vehicles respectively, which lead to practical results.

\section{Acknowledgement}

This paper is supported by the science and technology project of Guangdong province (2014A010106033); thenatural science foundation of Guangdong Province, the startup project of Guangdong province (2014A030310376);Guangdong Province Ordinary University Youth Innovative Talents Project (2014KQNCX201, 631051); The PhDStartup Fund of Natural Science Foundation of Guangdong Province (2014A030310376).

\section{References}

[1]Sun Jian, Yang Xiaoguang, A Study on Parameter Calibration of Microscopic Traffic Simulation Model System--Take the Application of VISSIM as an Example, Journal of Transport Information and Safety, 2004, 22 ( 03): 3-6. 
[2]Sun Jian, Yang Xiaoguang, Liu Dehao, A Study on Parameter Calibration of Microscopic Traffic Simulation Model System, Journal of Systems Engineering, 2007, 19 (01 ): 48-50.

[3]Zhou Jing, Chen Senfa, Simulation Study on Car-following Model of Microscopic Traffic Flows, Journal of Southeast University, 2004, 34 ( 04): 545-548.

[4]Wang Caixia, A Study on Lane-changing Model of Traffic Simulation, Jilin University, 2007.

[5]Zhang Qinghua, A Study on Calibration of VISSIM Microscopic Simulation Model, Beijing Jiaotong University, 2012. 